\title{
Publication trends of Allergy, Pediatric Allergy and Immunology, and Clinical and Translational Allergy journals: a MeSH term-based bibliometric analysis
}

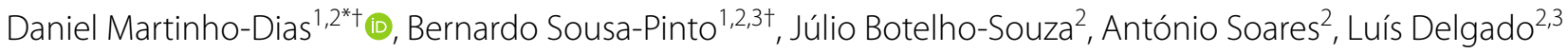 \\ and João Almeida Fonseca, ${ }^{1,2}$
}

\begin{abstract}
We performed a MeSH term-based bibliometric analysis aiming to assess the publication trends of EAACI journals, namely Allergy, Pediatric Allergy and Immunology (PAI) (from 1990 to 2015) and Clinical and Translational Allergy (CTA) (from its inception in 2011 to 2015). We also aimed to discuss the impact of the creation of CTA in the publication topics of Allergy and PAI. We analysed a total of 1973 articles and 23,660 MeSH terms. Most MeSH terms in the three journals fell in the category of "basic immunology and molecular biology" (BIMB). During the studied period, we observed an increase in the proportion of MeSH terms on BIMB, and a decreasing proportion of terms on allergic rhinitis and aeroallergens. The observed changes in Allergy and PAI publication topics hint at a possible impact from CTA creation.
\end{abstract}

Keywords: Bibliometrics, Medical subject headings, Research trends

\section{To the editor}

Bibliometric methods are used to quantitatively analyse published scientific literature for potential identification of relevant patterns $[1,2]$. We performed a MeSH termbased bibliometric analysis of publication topics of the three EAACI journals, namely Allergy, Pediatric Allergy and Immunology (PAI), and Clinical and Translational Allergy (CTA). We aimed to assess the evolution of publication trends in the fields of Allergy/Immunology, and to discuss whether the creation of CTA (in 2011) associated with changes in the publication topics by Allergy and PAI.

*Correspondence: danielmartinhodias@gmail.com

${ }^{\dagger}$ Daniel Martinho-Dias and Bernardo Sousa-Pinto contributed equally to this manuscript.

${ }^{1}$ MEDCIDS, Department of Community Medicine, Information and Health Decision Sciences, Faculty of Medicine, University of Porto, Rua Dr. Placido da Costa, 4200-450 Porto, Portugal

Full list of author information is available at the end of the article
We assessed original and review articles published in Allergy, PAI, and CTA. For each year between 1990 (first year of PAI) and 2015, we randomly selected and analysed $20 \%$ of Allergy publications and $40 \%$ of PAI publications. Additionally, we assessed all CTA publications until 2015. We retrieved the title, abstract and keywords of selected publications-keywords were converted into $\mathrm{MeSH}$ terms via Unified Medical Language System ${ }^{\circledR}$ terminology, while titles and abstracts were text-mined to obtain further MeSH terms. This was achieved using the online tool Syn4Data (http://www.syn4data.med.up.pt/). Two independent researchers verified the obtained results and excluded inadequate/wrongly attributed or generalist/uninformative $\mathrm{MeSH}$ terms (Additional file 1: Table 1).

For each journal, we obtained the frequency of publications to which each MeSH term was attributed. MeSH terms were categorised into nine "thematic" categories-(1) asthma and lower airways diseases, (2) allergic rhinitis and allergy to aeroallergens, (3) food allergy and 
nutrition, (4) drug allergy, (5) anaphylaxis and insect venom allergy, (6) skin and eye diseases, (7) diagnosis methods, (8) asthma and allergy therapy, and (9) basic immunology and molecular biology (BIMB). For each journal, we assessed the time trends of each category, performing a univariable logistic regression with time (in years) as independent variable.

We then compared the proportions of each category terms between the three journals. To address whether CTA had any influence in the topics published by Allergy and PAI, we compared MeSH terms proportions in these latter two journals prior and after 2011. Multivariable logistic regressions were performed for each category, adjusting for changes of the Editors-in-chief. Similar comparisons were performed concerning the proportions of publications assessing animal models.

We assessed 23,660 MeSH terms from 1973 articles. Most publications $(n=1001)$ and MeSH terms $(n=11,788)$ were from Allergy, followed by PAI (791 publications; 10,260 terms) and CTA (181 publications; 1612 terms). Allergy and CTA had significantly more publications with animal models (12.5 and $11.6 \%$, respectively) than PAI (5.6\%) ( $p<0.001$ and $p=0.003$, respectively). More post-2011 than pre-2011 Allergy publications assessed animal models (19.3 vs. $10.8 \% ; p=0.001$ ).

Within the three journals altogether, significant increases over time were observed for proportion of $\mathrm{MeSH}$ terms on "food allergy and nutrition" [OR 1.03 per year (95\% CI 1.02-1.04); $p<0.001$ ], "skin and eye diseases" [OR 1.04 per year (95\% CI 1.03-1.05); $p<0.001$ ], and "BIMB" [OR 1.01 per year (95\% CI 1.00-1.01); $p=0.003$ ] (Fig. 1a; Additional file 1: Table 2A). Most $\mathrm{MeSH}$ terms in all journals concerned BIMB, comprising $41.6 \%$ of Allergy terms, versus $33.5 \%$ of PAI $(p<0.001)$ and $29.5 \%$ of CTA $(p<0.001)$. In Allergy, more terms belonged to BIMB category after than before 2011 (51.0 vs. 38.8\%; $p<0.001$ ), even after adjustment for Editorial changes [OR 1.37 (95\% CI 1.09-1.73); $p=0.006$ ]. In PAI, the opposite trend was observed (35.8 post-2011 vs. $26.9 \%$ pre-2011; $p<0.001$ ), even after adjusting for the
Editors-in-chief [OR 0.62 (95\% CI 0.51-0.75); $p<0.001$ ] (Fig. 1b; Additional file 1: Table 2B).

On the contrary, post-2011 Allergy publications had lower proportion of terms on "diagnosis methods" (6.7 vs. $10.7 \% ; p<0.001$ ), "asthma and lower airways diseases" (7.4 vs. 9.4\%; $p=0.003$ ), and "allergic rhinitis and allergy to aeroallergens" (18.6 vs. $10.1 \% ; p<0.001)$; this latter decrease was significant even after adjusting for Editorial changes [OR 0.68 (95\% CI 0.49-0.95); $p=0.024]$. The proportion of "allergic rhinitis and allergy to aeroallergens" terms was also lower in postthan pre-2011 PAI articles (14.4 vs. 9.7\%; $p<0.001)$. Consistently, within the three journals altogether, there were significant decreases over time in the percentage of MeSH terms on "diagnosis methods" [OR 0.98 per year (95\% CI 0.98-0.99); $p<0.001]$ and "allergic rhinitis and allergy to aeroallergens" [OR 0.97 per year $(95 \% \mathrm{CI}$ $0.96-0.97) ; p<0.001]$.

In conclusion, this is the first bibliometric study assessing publication trends in the Allergy/Immunology field, although restricted to EAACI journals. Its keyword-based approach maximised the information extracted from publications' metadata [3]. The observed changes in Allergy and PAI post-2011 publication topics suggest a possible influence from CTA creation. Nevertheless, we did not assess other potentially relevant aspects, namely the existence and effect of other non-EAACI competing journals with a similar scope, the journals' impact factors, and the scientific and clinical background of the Editors-in-chief (we only adjusted for Editorial changes). Overall, during the studied period, we observed an increase in the proportion of MeSH terms on basic immunology, "skin and eye diseases" and "food allergy and nutrition", and a decreasing proportion of terms concerning "allergic rhinitis and aeroallergens" and "diagnosis methods". This analysis provides a starting point for discussing publication and research trends in the Allergy/Immunology field. Future studies should assess a larger set of journals in the field. 


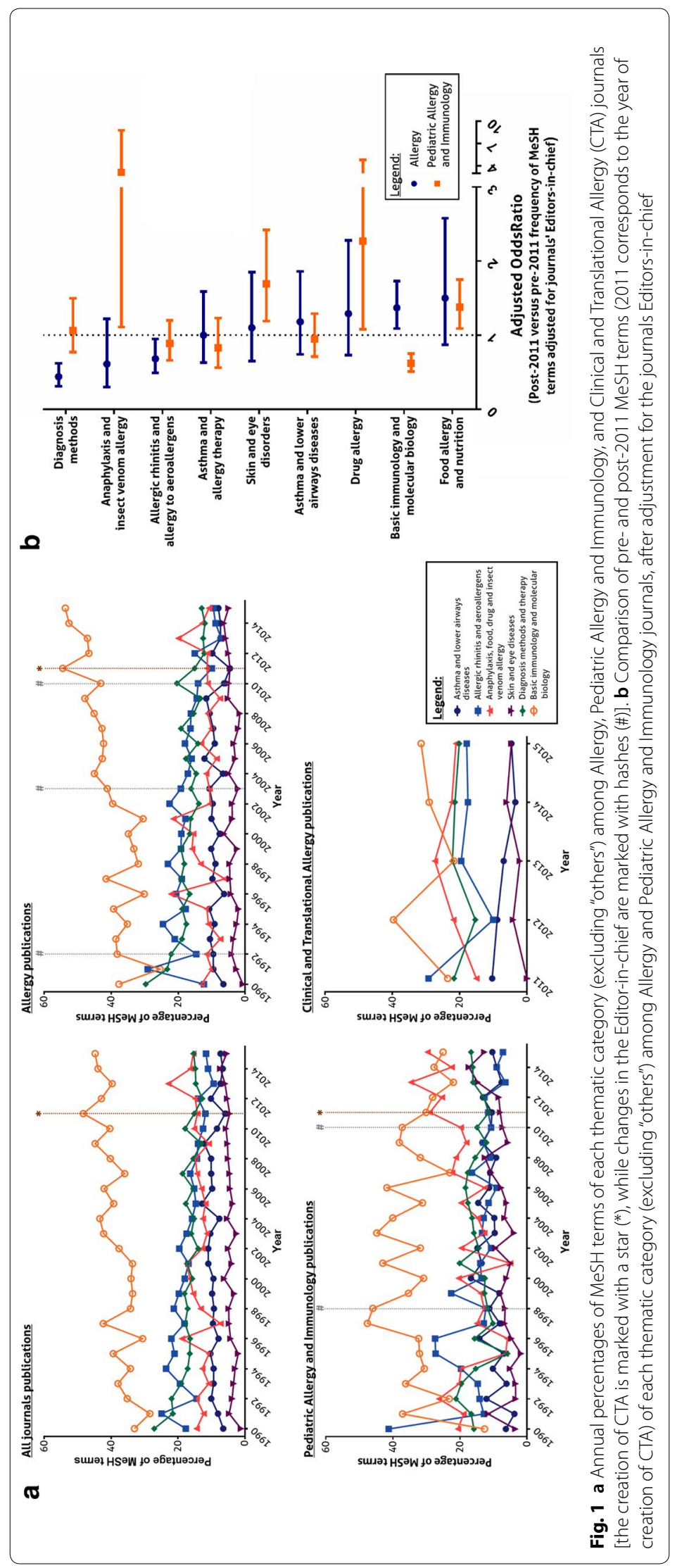




\section{Additional file}

Additional file 1: Table 1. List of excluded generalist MeSH terms in the context of an analysis in the field of allergy and immunology. Table $\mathbf{2}$. Temporal trends of MeSH terms of each thematic category (results obtained by univariable logistic regression, with the year of publication as independent variable) (A), and absolute and relative frequencies of MeSH terms of each thematic category (B) among Allergy, Pediatric Allergy and Immunology (PAI), and Clinical and Translational Allergy (CTA) journals.

\section{Abbreviations}

BIMB: basic immunology and molecular biology; CTA: Clinical and Translational Allergy; EAACl: European Academy of Allergy and Clinical Immunology; OR: odds ratio; PAl: Pediatric Allergy and Immunology.

\section{Authors' contributions}

DMD participated in the study design and development of the online tool Syn4Data, and analysed the data. BSP participated in the study design, analysed the data, and was a major contributor in writing the manuscript. JBS and AS participated in the development of the online tool Syn4Data, and were major contributors in revising the manuscript. LD participated in manuscript writing and critical revision of the manuscript. JAF participated in the study design, manuscript writing, and critical revision of the manuscript. All authors read and approved the final manuscript.

\section{Author details}

${ }^{1}$ MEDCIDS, Department of Community Medicine, Information and Health Decision Sciences, Faculty of Medicine, University of Porto, Rua Dr. Placido da Costa, 4200-450 Porto, Portugal. ${ }^{2}$ CINTESIS, Center for Health Technology and Services Research, Rua Dr. Placido da Costa, 4200-450 Porto, Portugal.

${ }^{3}$ Basic and Clinical Immunology Unit, Department of Pathology, Faculty of Medicine, University of Porto, Porto, Portugal.

\section{Acknowledgements}

None.

Preliminary results were presented in the EAACI Congress 2017 (17-21 June 2017, Helsinki, Finland), winning the Junior Members prize of the session "Education of health care professionals and patients".

\section{Competing interests}

The authors declare that they have no competing interests.

\section{Availability of data and materials}

The datasets used and/or analysed during the current study are available from the corresponding author on reasonable request.

\section{Consent for publication}

Not applicable.

Ethics approval and consent to participate

Not applicable.

\section{Funding}

Not applicable.

\section{Publisher's Note}

Springer Nature remains neutral with regard to jurisdictional claims in published maps and institutional affiliations.

Received: 16 November 2017 Accepted: 9 February 2018

Published online: 22 February 2018

\section{References}

1. Wallin JA. Bibliometric methods: pitfalls and possibilities. Basic Clin Pharmacol Toxicol. 2005;97(5):261-75.

2. Jayaratne YS, Zwahlen RA. The evolution of dental journals from 2003 to 2012: a bibliometric analysis. PLoS ONE. 2015;10(3):e0119503.

3. Chen G, Xiao L. Selecting publication keywords for domain analysis in bibliometrics: a comparison of three methods. J Informetr. 2016;10(1):212-23.

\section{Submit your next manuscript to BioMed Central and we will help you at every step:}

- We accept pre-submission inquiries

- Our selector tool helps you to find the most relevant journal

- We provide round the clock customer support

- Convenient online submission

- Thorough peer review

- Inclusion in PubMed and all major indexing services

- Maximum visibility for your research

Submit your manuscript at www.biomedcentral com/submit 